\title{
KTH
}

This is an accepted version of a paper published in IEEE Transactions on Automatic Control. This paper has been peer-reviewed but does not include the final publisher proof-corrections or journal pagination.

Citation for the published paper:

Sandberg, H. (2010)

"An Extension to Balanced Truncation With Application to Structured Model Reduction"

IEEE Transactions on Automatic Control, 55(4): 1038-1043

URL: http://dx.doi.org/10.1109/tac.2010.2041984

Access to the published version may require subscription.

(C) 2010 IEEE. Personal use of this material is permitted. Permission from IEEE must be obtained for all other uses, in any current or future media, including reprinting/ republishing this material for advertising or promotional purposes, creating new collective works, for resale or redistribution to servers or lists, or reuse of any copyrighted component of this work in other works.

Permanent link to this version:

http://urn.kb.se/resolve?urn=urn:nbn:se:kth:diva-19369

http://kth.diva-portal.org 


\section{An Extension to Balanced Truncation with Application to Structured Model Reduction}

\author{
Henrik Sandberg
}

\begin{abstract}
Balanced truncation is a standard method for model reduction, possessing many desirable properties such as preservation of model stability and a priori error bounds. Balanced truncation is conducted using controllability and observability Gramians. Generalized Gramians can be found by solving a set of linear matrix inequalities. In this paper, we show that these linear matrix inequalities can be extended so that the number of decision variables are at least doubled, leading to the concept of extended Gramians. Herein it is shown that the desirable properties of balanced truncation also hold with extended Gramians. The extended Gramians are especially useful for improving error bounds and for models possessing additional structural constraints, as is demonstrated by means of examples herein.
\end{abstract}

Index Terms-Error bounds; LMIs; Model order reduction; Structure constraints.

\section{INTRODUCTION}

Model (order) reduction concerns the systematic simplification of complex models. Although this is a topic that has received a great deal of attention since the early 1980's, fundamental problems still remain to be solved. In particular, most available methods that are guaranteed to deliver good approximations only apply to linear systems, and these techniques usually do not preserve the topological structures of the original models. In this paper, we propose an extension to the well-known balanced truncation method, thereby offering new degrees of freedom which can be used both to lower error bounds and to enforce structural constraints.

Balanced truncation is a standard tool to reduce the order of linear systems. This method was introduced in [1], [2] and it was later revealed in [3] that the stability of models is preserved, and in [4], [5] that simple a priori error bounds hold. These results were all shown for continuous-time models. In discrete time, the corresponding results were shown in [6]. To apply balanced truncation, both controllability and observability Gramians are needed (herein called regular Gramians). These are obtained from solving two Lyapunov equations. In [7], it was shown that if the Lyapunov equations are replaced by linear matrix inequalities (LMIs), generalized Gramians can be defined and the error bounds in [6] can be improved by enforcing multiplicity of Hankel singular values. Generalized Gramians have later been used also to characterize the solution to the optimal $H_{\infty}$ model reduction problem [8]-[10], and for model reduction of multidimensional and uncertain systems [10], [11], for example. In this paper, we build upon the idea of generalized Gramians and introduce even more flexible extended Gramians and inequalities. The increased flexibility can be used to further improve error bounds, and to more effectively enforce topological constraints in model reduction ("structured model reduction"). Generalized Gramians have earlier also been used for structured model reduction, see [12]-[14].

The extension of linear matrix inequalities and introduction of new degrees of freedom as applied in this paper was first proposed in [15], [16]. In [15], [16] the applications were stability analysis and controller synthesis for parameter-dependent models. The same type of extension was also used in [17] to construct distributed estimators.

H. Sandberg is at Royal Institute of Technology (KTH), School of Electrical Engineering, Automatic Control, SE-100 44 Stockholm, Sweden. Phone: +468-7907427, Email: hsan@ee. kth. se, Fax: +46-8-7907329.

This work was supported by the Swedish Foundation for Strategic Research and the Swedish Research Council.
In [18], the extension was first used for model reduction. In this paper, more general results showing how extended Gramians can be used for model comparison, and a new application on structured model reduction are presented.

The organization of the paper is as follows. In Section II, we introduce the model structures and define what we mean by model reduction. In Section III, generalized and extended Gramians are defined, and their relation is discussed. In Section IV, it is shown how the extended Gramians can be used to prove approximation error bounds for truncated models and how they can be balanced. Finally, in Section V extended balanced truncation is applied to two examples.

\section{Preliminaries and Problem Formulation}

We consider stable linear finite-dimensional discrete-time systems $G$ of order $n$ in this paper, with realization

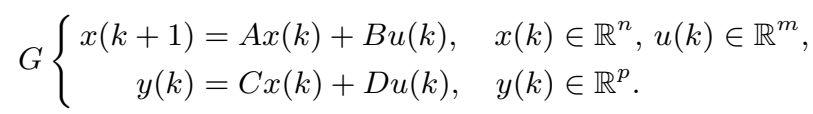

For simplicity, the time index $k$ is often left out in the notation, and the notation $x^{+}:=x(k+1), x:=x(k), u:=u(k)$ etc. is used. Thus, the realization can be written as

$$
G\left\{\begin{array}{rlrl}
x^{+} & =A x+B u, & & x \in \mathbb{R}^{n}, u \in \mathbb{R}^{m}, \\
y & =C x+D u, & y \in \mathbb{R}^{p} .
\end{array}\right.
$$

The transfer function of $G$, denoted by $G(z)$, is defined by $G(z):=D+C(z I-A)^{-1} B$ and the $H_{\infty}$-norm by $\|G\|_{\infty}:=$ $\sup _{z \in \mathbb{C} \backslash \overline{\mathbb{D}}}|G(z)|$, where $\overline{\mathbb{D}}$ is the closed unit disc in the complex plane $\mathbb{C}$. For stable systems $G$ it holds that $\|G\|_{\infty}<\infty$. With $\|u\|_{\left[k_{1}, k_{2}\right]}$, we mean $\|u\|_{\left[k_{1}, k_{2}\right]}^{2}:=\sum_{k=k_{1}}^{k_{2}} u(k)^{T} u(k)$ and $\|u\|:=\|u\|_{[0, \infty]}$. Square-summable sequences, $\|u\|<\infty$, belong to the Hilbert space $\ell_{2}$. It is well-known that $\|G\|_{\infty}=\sup _{u \in \ell_{2} \backslash\{0\}}\|y\| /\|u\|$, where $y, u$ satisfy the equations of the realization of $G$.

The model-reduction problem is to find a new linear system $\hat{G}$ with order $r<n$, such that $\|G-\hat{G}\|_{\infty}$ is small. In this paper, this is done by truncating the realization of $G$. We use the partitioning

$$
\begin{gathered}
A=\left[\begin{array}{ll}
A_{11} & A_{12} \\
A_{21} & A_{22}
\end{array}\right], B=\left[\begin{array}{l}
B_{1} \\
B_{2}
\end{array}\right], C=\left[\begin{array}{ll}
C_{1} & C_{2}
\end{array}\right], \\
A_{11} \in \mathbb{R}^{r \times r}, B_{1} \in \mathbb{R}^{r \times m}, C_{1} \in \mathbb{R}^{p \times r} .
\end{gathered}
$$

A candidate approximation $\hat{G}$ is given by

$$
\hat{G}\left\{\begin{aligned}
\hat{x}_{1}^{+} & =A_{11} \hat{x}_{1}+B_{1} u, & \hat{x}_{1} & \in \mathbb{R}^{r}, u \in \mathbb{R}^{m}, \\
\hat{y} & =C_{1} \hat{x}_{1}+D u, & \hat{y} & \in \mathbb{R}^{p} .
\end{aligned}\right.
$$

The main benefit of the balanced-truncation method is that it gives good state coordinates $x$ for truncation, and reveals how the approximation order $r$ can be chosen such that $\|G-\hat{G}\|_{\infty}$ is guaranteed to be small. As we shall see, extended balanced truncation has the same benefits, and allows for some additional degrees of freedom.

\section{EXTENDED LYAPUNOV INEQUALITIES AND EXTENDED GRAMIANS}

In this section, the extended Lyapunov inequalities and the extended Gramians are introduced and interpreted. We start by defining the generalized Gramians and Lyapunov inequalities.

As is well known, the controllability and observability Lyapunov linear matrix inequalities,

$$
\begin{aligned}
& P-A P A^{T}-B B^{T} \geq 0, \\
& Q-A^{T} Q A-C^{T} C \geq 0,
\end{aligned}
$$


have symmetric and positive semidefinite solutions in $\mathbb{R}^{n \times n}$,

$$
P=P^{T} \geq 0, \quad Q=Q^{T} \geq 0,
$$

if the realization of $G$ is asymptotically stable $(\rho(A)<1)$. Solutions $P, Q$ are called generalized controllability and observability Gramians, respectively, and are easily computed using convex optimization software, for example [19]. The extended controllability and observability Lyapunov linear matrix inequalities are given by

$$
\begin{gathered}
{\left[\begin{array}{ccc}
P & A R & B \\
R^{T} A^{T} & R+R^{T}-P & 0 \\
B^{T} & 0 & I
\end{array}\right] \geq 0,} \\
{\left[\begin{array}{ccc}
S+S^{T}-Q & S A & 0 \\
A^{T} S^{T} & Q & C^{T} \\
0 & C & I
\end{array}\right] \geq 0,}
\end{gathered}
$$

with extended controllability Gramian $(P, R)$ and extended observability Gramian $(Q, S)$. The extended Gramians have two components each, and $P=P^{T}, Q=Q^{T} \in \mathbb{R}^{n \times n}$ are symmetric and $R, S \in \mathbb{R}^{n \times n}$ are possibly non-symmetric. The variables $R, S$ are the extra degrees of freedom as mentioned previously. The introduction of new variables in linear matrix inequalities in this way was first done by De Oliviera et al. in [15], [16]. Feasibility of the inequalities are in fact equivalent, see Theorem 1 below. Such equivalences were also derived in the references [15], [16].

Remark 1: Note that generalized and extended Gramians are not unique, and not all feasible solutions will yield reasonable model approximations. To find generalized Gramians that yield good and even optimal model approximations, various additional optimization criteria and constraints have been proposed. In [8], [9], it is shown that if certain rank constraints are satisfied by generalized Gramians, optimal $H_{\infty}$-approximations are obtained. The rank constraints are hard to enforce numerically, however. In Section V, we will simply minimize the traces of the generalized and extended Gramians subject to (2)-(3) and (5)-(6). To minimize the trace is a heuristic with certain desirable properties that was suggested in [20]. For example, $P, Q$ of minimum traces subject to (2)-(3) are identical to the regular Gramians, and then give necessary and sufficient conditions for controllability and observability.

Theorem 1: The inequalities (2)-(4) have solutions $P=P^{T}, Q=$ $Q^{T}$ if, and only if, the inequalities (5)-(6) have solutions $P=$ $P^{T}, Q=Q^{T}$ and $R, S$.

Proof: We prove the observability case here. The controllability case is similar. For strict inequalities, the result is shown in Theorem 1 in [16]. Since the derivation helps to understand the roles of $P, Q$ and $R, S$, the result is shown next. Sufficiency: If (3) and (4) hold, then

$$
\begin{aligned}
{\left[\begin{array}{cc}
Q & 0 \\
0 & Q-A^{T} Q A-C^{T} C
\end{array}\right] \geq 0 } & \Leftrightarrow\left[\begin{array}{cc}
Q & Q A \\
A^{T} Q & Q-C^{T} C
\end{array}\right] \geq 0 \\
& \Leftrightarrow\left[\begin{array}{ccc}
Q & Q A & 0 \\
A^{T} Q & Q & C^{T} \\
0 & C & I
\end{array}\right] \geq 0
\end{aligned}
$$

using Schur complements [21]. Hence, the inequality (6) holds if we use $S=Q$. Necessity: Assume the (6) has a solution $Q=Q^{T}$ and $S$. Again using Schur complements, (6) is equivalent to

$$
\left[\begin{array}{cc}
S+S^{T}-Q & S A \\
A^{T} S^{T} & Q-C^{T} C
\end{array}\right] \geq 0 .
$$

Multiplying this inequality from the left and the right with proper matrices gives

$$
\begin{array}{r}
{\left[\begin{array}{c}
-A \\
I
\end{array}\right]^{T}\left[\begin{array}{cc}
S+S^{T}-Q & S A \\
A^{T} S^{T} & Q-C^{T} C
\end{array}\right]\left[\begin{array}{c}
-A \\
I
\end{array}\right] \geq 0} \\
\quad \Rightarrow Q-A^{T} Q A-C^{T} C \geq 0 .
\end{array}
$$

From the $(2,2)$ block of (6) it is also seen that $Q \geq 0$. Hence (3) and the right inequality in (4) hold.

From the proof, it is seen that if the extended inequalities (5)(6) are solved then the $P, Q$ components of the extended Gramians can always be used as generalized Gramians that solve (2)-(4). This justifies that the same notation is used for the first components of the extended Gramians and for the generalized Gramians. If the Lyapunov inequalities (2)-(3) are solved, then the generalized Gramians $P, Q$ can always be used to construct extended Gramians $(P, P)$ and $(Q, Q)$ that solve (5)-(6). However, this does not yield new information. The concept presented herein is to not choose $R=P, S=Q$ and instead utilize the extra degrees of freedom.

\section{Model COMPARISON AND EXTEnded BALANCED TRUNCATION}

In this section, we use the extended Lyapunov inequalities to compare models and to extend the balanced truncation method.

\section{A. Model Comparison}

Consider the two realizations

$$
\begin{aligned}
G\left\{\begin{aligned}
x^{+} & =A x+B u, \quad x(0)=x_{0}, \\
y & =C x+D u,
\end{aligned}\right. \\
G_{r}\left\{\begin{aligned}
x_{r}^{+} & =A x_{r}+B u+v, \quad x_{r}(0)=x_{r 0}, \\
y_{r} & =C x_{r}+D u .
\end{aligned}\right.
\end{aligned}
$$

The model $G_{r}$ is identical to $G$ apart from a perturbation signal $v(k) \in \mathbb{R}^{n}$ and a potentially different initial state. The same input $u$ is applied to both models. Using the the extended Gramians, we can quantify the differences between the two models $G$ and $G_{r}$. Using Schur complements on the extended Lyapunov inequality (6), and multiplying it from left and right with a properly chosen combination of the state vectors of $G$ and $G_{r}$, we obtain

$$
\begin{gathered}
{\left[\begin{array}{c}
-\left(x-x_{r}\right)^{+} \\
x-x_{r}
\end{array}\right]^{T}\left[\begin{array}{cc}
S+S^{T}-Q & S A \\
A^{T} S^{T} & Q-C^{T} C
\end{array}\right]\left[\begin{array}{c}
-\left(x-x_{r}\right)^{+} \\
x-x_{r}
\end{array}\right] \geq 0} \\
\Leftrightarrow\left(x-x_{r}\right)^{T} Q\left(x-x_{r}\right)-\left(x-x_{r}\right)^{+T} Q\left(x-x_{r}\right)^{+} \\
-2\left(x-x_{r}\right)^{+T} S v \geq\left|y-y_{r}\right|^{2},
\end{gathered}
$$

where $x^{+T}:=x(k+1)^{T}$. Summing this inequality over $k \in[0, T]$, we obtain the following proposition.

Proposition 1: Suppose the model $G$ has an extended observability Gramian $(Q, S)$. Then the outputs of $G$ and $G_{r}$ in (7) satisfy the bound

$$
\begin{array}{rl}
\left\|y-y_{r}\right\|_{[0, T]}^{2} \leq\left(x_{0}-x_{r, 0}\right)^{T} & Q\left(x_{0}-x_{r, 0}\right) \\
& -2 \sum_{k=0}^{T}\left[\left(x-x_{r}\right)(k+1)\right]^{T} S v(k),
\end{array}
$$

for all $T \geq 0$.

When there is no perturbation, $v=0$, then $G$ and $G_{r}$ only differ in the initial state and the norm of the output error only depends on $Q$, $x_{0}$, and $x_{r, 0}$, as is well known from normal observability analysis, see for example [22]. The component $S$ of the extended Gramian only shows up when there is a non-zero perturbation signal $v$. The bound shows that the sum containing $v(k)$ and $x(k+1)-x_{r}(k+1)$ cannot be made arbitrarily positive. When $T=1$ and $G, G_{r}$ are 
initially at rest, it follows that $\left|y(1)-y_{r}(1)\right|^{2} \leq 2 v(0)^{T} S v(0)$. This is no contradiction since $S$ is positive semidefinite. It is also seen that if the perturbation signal falls in the nullspace of $S$, it does not cause an output error $y-y_{r}$.

There is a dual result in the controllability case. Without loss of generality, assume the component $R$ of the extended controllability Gramians is invertible. If $R$ is not invertible it means that there is an uncontrollable state, which can be removed from the model. This yields a new invertible $R$. Let us define $\tilde{R}:=R^{-1}, \tilde{P}:=\tilde{R}^{T} P \tilde{R}$. We can multiply the extended controllability Lyapunov inequality in (5) from the left with $\operatorname{diag}\{\tilde{R}, \tilde{R}, I\}^{T}$ and from the right with $\operatorname{diag}\{\tilde{R}, \tilde{R}, I\}$. If this new inequality is multiplied from the left and right with a properly chosen combination of state vectors and input from the models $G$ and $G_{r}$ in (7), we obtain

$$
\begin{aligned}
{\left[\begin{array}{c}
-\left(x+x_{r}\right)^{+} \\
x+x_{r} \\
2 u
\end{array}\right]^{T} } & {\left[\begin{array}{ccc}
\tilde{P} & \tilde{R}^{T} A & \tilde{R}^{T} B \\
A^{T} \tilde{R} & \tilde{R}+\tilde{R}^{T}-\tilde{P} & 0 \\
B^{T} \tilde{R} & 0 & I
\end{array}\right]\left[\begin{array}{c}
-\left(x+x_{r}\right)^{+} \\
x+x_{r} \\
2 u
\end{array}\right] \geq 0 } \\
& \Leftrightarrow\left(x+x_{r}\right)^{T}\left(\tilde{R}+\tilde{R}^{T}-\tilde{P}\right)\left(x+x_{r}\right) \\
& -\left(x+x_{r}\right)^{+T}\left(\tilde{R}+\tilde{R}^{T}-\tilde{P}\right)\left(x+x_{r}\right)^{+} \\
& +2\left(x+x_{r}\right)^{+T} \tilde{R}^{T} v+4|u|^{2} \geq 0 .
\end{aligned}
$$

Summing this inequality over the time interval $k \in[0, T]$, we obtain the following proposition.

Proposition 2: Suppose the model $G$ is controllable and has an extended controllability Gramian $(P, R)$. Then if $x_{0}=x_{r, 0}=0$, the states of $G$ and $G_{r}$ in (7) satisfy the bound

$$
\begin{aligned}
{\left[\left(x+x_{r}\right)(T+1)\right]^{T}\left(\tilde{R}+\tilde{R}^{T}-\tilde{P}\right)\left[\left(x+x_{r}\right)(T+1)\right] } \\
\quad-2 \sum_{k=0}^{T}\left[\left(x+x_{r}\right)(k+1)\right]^{T} \tilde{R}^{T} v(k) \leq 4\|u\|_{[0, T]}^{2},
\end{aligned}
$$

for all $T \geq 0$, where $\tilde{R}:=R^{-1}$ and $\tilde{P}:=\tilde{R}^{T} P \tilde{R}$.

The interpretation of Proposition 2 is less clear than the interpretation of Proposition 1. Note, however, that the component $R$ can always be choosen such that $\tilde{R}=P^{-1}$. If there is no perturbation signal, $v=0$, then Proposition 2 reduces to $x(T+1)^{T} P^{-1} x(T+1) \leq$ $\|u\|_{[0, T]}^{2}$, which also follows from normal controllability analysis, for example see [22]. Hence, Proposition 2 gives an extension to known results. The propositions are next applied to truncated models, when $G_{r}=\hat{G}$, but they can also be used in other situations.

\section{B. Model Truncation and Balanced Extended Gramians}

First notice that with a particular choice of perturbation signal $v$, the model $G_{r}$ and the truncated model $\hat{G}$ in (1) are identical.

Lemma 1: Partition the state vector of $G_{r}$ in (7) into $x_{r}^{T}=$ $\left[\begin{array}{ll}x_{r, 1}^{T} & x_{r, 2}^{T}\end{array}\right]$, where $x_{r, 1}(k) \in \mathbb{R}^{r}$ and $x_{r, 2}(k) \in \mathbb{R}^{s}, s:=n-r$. Suppose the perturbation signal is chosen as $v(k)^{T}=\left[\begin{array}{ll}0 & -z(k)^{T}\end{array}\right]$, $z(k):=A_{21} x_{r, 1}(k)+B_{2} u(k)$, and $x_{r 0}=0$ and $\hat{x}(0)=0$. Then the outputs from $\hat{G}$ in (1) and $G_{r}$ in (7) are identical: $y_{r}(k)=\hat{y}(k)$, and $x_{r, 2}(k)=0, k \geq 0$.

Let us next make an assumption on the $R, S$ components of the extended Gramians. Suppose they have the block-diagonal structure

$$
R=\left[\begin{array}{cc}
R_{1} & 0 \\
0 & \sigma I_{s}
\end{array}\right], \quad S=\left[\begin{array}{cc}
S_{1} & 0 \\
0 & \sigma I_{s}
\end{array}\right],
$$

where $\sigma \in \mathbb{R}$ is a positive real number. Below it is shown how it is possible to fulfill this assumption. No special structure is assumed for the $P, Q$ components, apart from them being symmetric positive semidefinite matrices. Using these inequalities we have the following lemma that bounds the input-output approximation error.
Lemma 2: Suppose $G$ has extended Gramians $(P, R)$ and $(Q, S)$, where the $R, S$ components have the structure (8). If $G$ and the truncated model $\hat{G}$ initially are at rest, then it holds that $\| y-$ $\hat{y}\left\|_{[0, T]} \leq 2 \sigma\right\| u \|_{[0, T]}$, for all $T \geq 0$. That is $\|G-\hat{G}\|_{\infty} \leq 2 \sigma$.

Proof: Multiply the bound in Proposition 2 with $\sigma^{2}$, and add it to the bound in Proposition 1. If we use the choice of $v$ in Lemma 1 (so that $\hat{G}=G_{r}$ and $x_{r, 2}=0$ ) and the structure (8), we note that the sums containing the sign-indefinite terms $x_{2}^{+T} z$ cancel. All the remaining terms are positive and the result follows.

Hence, if we truncate states and have block-diagonal $R, S$ components, then the input-output approximation error is easily bounded. As we see next, the result can be applied recursively.

Lemma 3: Suppose $G$ has extended Gramians in the form

$$
\begin{aligned}
& P=\left[\begin{array}{ll}
P_{11} & P_{12} \\
P_{12}^{T} & P_{22}
\end{array}\right], R=\left[\begin{array}{cc}
R_{1} & 0 \\
0 & \sigma I_{s}
\end{array}\right], \\
& Q=\left[\begin{array}{ll}
Q_{11} & Q_{12} \\
Q_{12}^{T} & Q_{22}
\end{array}\right], S=\left[\begin{array}{cc}
S_{1} & 0 \\
0 & \sigma I_{s}
\end{array}\right],
\end{aligned}
$$

where $P_{11}, R_{1}, Q_{11}, S_{1} \in \mathbb{R}^{r \times r}, P_{22}, Q_{22} \in \mathbb{R}^{s \times s}$. Then the truncated system $\hat{G}$ has the extended Gramians $\left(P_{11}, R_{1}\right)$ and $\left(Q_{11}, S_{1}\right)$.

Proof: See proof of Lemma 2 in [18].

Next we justify the assumption (8). Let us consider coordinate transformations $\bar{x}=T x$, where $T$ is invertible. We know that the realization of the model $G$ transforms as $\bar{A}=T A T^{-1}, \bar{B}=T B$, $\bar{C}=C T^{-1}, \bar{D}=D$. The extended Gramians transformation is shown in the next proposition.

Proposition 3: Under invertible coordinate transformations $\bar{x}=$ $T x$, the extended Gramians transform as $\bar{P}=T P T^{T}, \bar{R}=T R T^{T}$, $\bar{Q}=T^{-T} Q T^{-1}$, and $\bar{S}=T^{-T} S T^{-1}$.

Proof: Replace $A, B, C$ in (6) with $T^{-1} \bar{A} T, T^{-1} \bar{B}$, and $\bar{C} T$. If (6) is multiplied with $\operatorname{diag}\left\{T^{T}, T^{T}, I\right\}$ from the right, and with the transpose from the left, then we can identify $\bar{P}$ and $\bar{R}$. A similar technique is used to prove the observability case.

The $R, S$ components transform just as the Gramians $P$ and $Q$. In particular, the eigenvalues $\lambda_{i}(P Q)=\lambda_{i}(\bar{P} \bar{Q})$ and $\lambda_{i}(R S)=$ $\lambda_{i}(\bar{R} \bar{S})$, are invariant under coordinate transformations. The numbers $\sigma_{i}=\sqrt{\lambda_{i}(P Q)}$ are often called generalized Hankel singular values. It is well known that there is a coordinate transformation $T$ that makes $\bar{P}$ and $\bar{Q}$ equal and diagonal [2]. Since $R$ and $S$ transform in the same way, one could hope that $\bar{R}$ and $\bar{S}$ can also be made equal and diagonal. However, if $R$ and $S$ are not symmetric, such a coordinate transformation may not exist. But we can always sacrifice some degrees of freedom in $R$ and $S$ and make them symmetric.

Proposition 4: Let the $R, S$ components of the extended Gramians be symmetric. Then there exists a coordinate transformation $\bar{x}=T x$ such that

$$
\begin{gathered}
\bar{R}=\bar{S}=\Sigma_{e}=\operatorname{diag}\left\{\sigma_{e, 1} I_{s_{1}}, \ldots, \sigma_{e, N} I_{s_{N}}\right\}, \\
\sigma_{e, 1}>\ldots>\sigma_{e, N} \geq 0
\end{gathered}
$$

where $\sigma_{e, i}:=\sqrt{\lambda_{i}(R S)}$ are the extended Hankel singular values. Here $s_{i}, i=1, \ldots, N$ and $s_{1}+\ldots+s_{N}=n$, is the multiplicity of $\sigma_{e, i}$.

Proof: A balancing transformation $T$ can be constructed just as for regular Gramians but using the symmetric $R$ and $S$, see [2].

The extended Gramians are called balanced extended Gramians if the $R, S$ components have the diagonal form $\Sigma_{e}$. The main theorem of the paper can now be stated.

Theorem 2: Suppose that $G$ has balanced extended Gramians 
$\left(P, \Sigma_{e}\right)$ and $\left(Q, \Sigma_{e}\right)$ where $\Sigma_{e}=\operatorname{diag}\left\{\Sigma_{e, 1}, \Sigma_{e, 2}\right\}$ and

$$
\begin{aligned}
& \Sigma_{e, 1}=\operatorname{diag}\left\{\sigma_{e, 1} I_{s_{1}}, \ldots, \sigma_{e, l} I_{s_{l}}\right\}, \\
& \Sigma_{e, 2}=\operatorname{diag}\left\{\sigma_{e, l+1} I_{s_{l+1}}, \ldots, \sigma_{e, N} I_{s_{N}}\right\}, \quad r=s_{1}+\ldots+s_{l} .
\end{aligned}
$$

Then the truncated $r$-th order system $\hat{G}$ has balanced extended Gramians $\left(P_{11}, \Sigma_{e, 1}\right)$ and $\left(Q_{11}, \Sigma_{e, 1}\right)$, and

$$
\|G-\hat{G}\|_{\infty} \leq 2 \sum_{i=l+1}^{N} \sigma_{e, i} .
$$

Proof: The theorem follows by iteratively applying Lemma 2 and Lemma 3 to the balanced realization. The error bound then follows from the triangle inequality.

It is the $R, S$ components of the extended Gramians that provide the input-output error bound in Theorem 2. Note that if we sacrifice the extra degrees of freedom provided by $R, S$ and enforce $R=P, S=Q$ and balance all components, the result reduces to generalized balanced truncation [7] since $\sigma_{e, i}=\sigma_{i}$. Thus we can expect that the reduced models coming from extended balanced truncation are at least as good as those coming from generalized balanced truncation.

The non-uniqueness of the extended Gramians can be used to enforce multiplicity greater than one for the extended Hankel singular values. If $s_{N}=n-r$, the bound in Theorem 2 becomes $2 \sigma_{e, N}$. A similar idea was used in [7] to show that generalized Gramians can provide better error bounds than regular Gramians. In fact, if there exists an $r$-th order model $\hat{G}$ such that $\|G-\hat{G}\|_{\infty} \leq \epsilon$, then there exist generalized Gramians with a generalized Hankel singular value equal to $\epsilon$ and of multiplicity $n-r$, see [8]-[10]. A similar statement holds for extended Gramians since we can use $R=P, S=Q$. These Gramians are hard to find numerically because of rank constraints, see for example [9], and we do not further pursue this idea here. Instead we evaluate how a simpler convex trace criterion for $R, S$ performs, see Remark 1 and Examples 1-2 below.

\section{Model Reduction ExAmples}

In this section, two examples that illustrate the benefits of extended balanced truncation are presented. Further examples are given in [18].

Example 1: Here we apply extended balanced truncation to a set of randomly generated systems of various order $n, 10 \leq n \leq 30$, and compare the result with generalized balanced truncation. The generalized Gramians are computed by solving the LMIs

$\min \operatorname{Tr} P$ and $\min \operatorname{Tr} Q$; subject to (2)-(3),

and the extended Gramians are computed by solving the LMIs

$$
\min \operatorname{Tr} R \text { and } \min \operatorname{Tr} S \text {; subject to (5)-(6) }
$$$$
\text { and } R=R^{T}, S=S^{T} \text {, }
$$

using the solver SeDuMi [19]. Minimization of the trace is a heuristic suggested in [20] to make the Gramians small. Note that the solution of (9) coincides with the regular Gramians since there are no structure constraints on $P$ and $Q$. Thus generalized and regular balanced truncation are identical in these tests. For each order $n, 20$ random systems $G$ are created and are scaled such that $\rho(A) \leq 0.99$ and $G(1)=1000$. In total 420 systems are approximated. The systems are approximated with first-order systems $\hat{G}$, i.e. $n-1$ states are removed. In Table I, the arithmetic means of the error bounds, actual approximation errors, and the computation times are reported for the cases $n=10,15,20,25,30$.

The approximations $\hat{G}$ given by extended and regular truncation are almost identical in these tests. On average, extended truncation yields $0.1 \%$ smaller approximation errors $\|G-\hat{G}\|_{\infty}$, but this is of no practical significance. Therefore there is only one column of approximation errors in Table I.

The real advantage of extended truncation is seen in the upper error bounds $2 \sum_{i=2}^{n} \sigma_{e, i}$ and $2 \sum_{i=2}^{n} \sigma_{i}$ in Table I. The generalized and the regular Hankel singular values of $G$ are identical and are denoted by $\sigma_{i}$ here. The simple trace criterion used to fix $R, S$ yields on average $35 \%$ better error bounds than regular balanced truncation. This improvement does not seem to depend on the model order $n$. A lower error bound is given by $\sigma_{2}$, and it is seen that the approximations are quite close to this theoretical limit.

In these tests, it took on average 2.3 times more time to solve (10) than it took to solve (9). A worst-case estimate of the number of arithmetic operations needed to solve Lyapunov inequalities is $O\left(l^{2.75}\right)$, where $l$ is the number of optimization variables, see [21]. Since extended Gramians have twice as many variables as generalized Gramians, we can expect about 7 times longer computation, but this estimate seems quite conservative.

The example shows that extended balanced truncation gives better error bounds than generalized and regular balanced truncation when the trace criterium is used. The price is a longer computation time. The approximations are also essentially identical to the ones obtained with regular balanced truncation. It is an interesting open problem to characterize when the two methods must give essentially identical approximations. It should be noted that regular Gramians can be computed faster than generalized Gramians by solving Lyapunov equations using $O\left(n^{3}\right)$ operations. However, regular Gramians cannot be used when there are additional structure constraints such as in the next example.

Example 2: Here we reduce a model with a topological structure constraint. Methods for solving such problems have been considered also in for example [12], [23] and in [14] where generalized Gramians were used. Here it is shown how extended Gramians can be applied.

Consider a negative feedback interconnection with transfer function (complex argument $z$ is left out to simplify notation)

$$
T\left(k, G^{1}, G^{2}\right):=\frac{1}{1+k G^{1} G^{2}}\left(\begin{array}{cc}
G^{1} & -k G^{1} G^{2} \\
k G^{1} G^{2} & k G^{2}
\end{array}\right),
$$

consisting of two systems $G^{1}, G^{2}$ and a scalar feedback gain $k \geq$ 0 . The problem is to find reduced-order models $\hat{G}^{1}, \hat{G}^{2}$ such that $\left\|T\left(k, G^{1}, G^{2}\right)-T\left(k, \hat{G}^{1}, \hat{G}^{2}\right)\right\|_{\infty}$ is small. Let the vectors $x^{1}(k) \in$ $\mathbb{R}^{n^{1}}, x^{2}(k) \in \mathbb{R}^{n^{2}}$ be the states of the subsystems $G^{1}, G^{2}$. The feedback system $T\left(k, G^{1}, G^{2}\right)$ can then be realized with a $n=n^{1}+$ $n^{2}$ dimensional state vector $x^{T}=\left[\begin{array}{ll}\left(x^{1}\right)^{T} & \left(x^{2}\right)^{T}\end{array}\right]$ and conformably structured matrices

$$
\begin{array}{rlr}
A=\left[\begin{array}{ll}
A^{11} & A^{12} \\
A^{21} & A^{22}
\end{array}\right], & B=\left[\begin{array}{cc}
B^{1} & 0 \\
0 & B^{2}
\end{array}\right], \\
C=\left[\begin{array}{cc}
C^{1} & 0 \\
0 & C^{2}
\end{array}\right], & D=\left[\begin{array}{cc}
D^{1} & 0 \\
0 & D^{2}
\end{array}\right] .
\end{array}
$$

If regular model reduction is applied to $T\left(k, G^{1}, G^{2}\right)$, the feedback structure is typically lost in the reduced model. To maintain the feedback structure, the reduced state vector should be in the form $\hat{x}^{T}=\left[\begin{array}{ll}\left(\hat{x}^{1}\right)^{T} & \left(\hat{x}^{2}\right)^{T}\end{array}\right], \hat{x}^{1}(k) \in \mathbb{R}^{r^{1}}, \hat{x}^{2}(k) \in \mathbb{R}^{r^{2}}, r^{1}+r^{2}=r$, such that $\hat{x}^{1}$ is only a function of $x^{1}$, and $\hat{x}^{2}$ is only a function of $x^{2}$.

One way to preserve the structure in the reduced model is to apply generalized balanced truncation to the model (11) and solve the LMIs

$$
\begin{aligned}
& \min \operatorname{Tr} P \text { and } \min \operatorname{Tr} Q ; \text { subject to (2)-(3) } \\
& \text { and } P=\operatorname{diag}\left\{P^{1}, P^{2}\right\}, Q=\operatorname{diag}\left\{Q^{1}, Q^{2}\right\},
\end{aligned}
$$

where $P^{k}, Q^{k} \in \mathbb{R}^{n^{k} \times n^{k}}, k=1,2$, as suggested in for example [12], [14]. Each subsystem can then be balanced and truncated separately 
TABLE I

RESULTS FROM THE NUMERICAL TESTS OF GENERALIZED AND EXTENDED BALANCED TRUNCATION IN EXAMPLE 1.

\begin{tabular}{ccccccc}
\hline$n$ & $\left\langle\sigma_{2}\right\rangle$ & $\left\langle\|G-\hat{G}\|_{\infty}\right\rangle$ & $\left\langle 2 \sum_{i=2}^{n} \sigma_{i}\right\rangle$ & $\left\langle 2 \sum_{i=2}^{n} \sigma_{e, i}\right\rangle$ & $\langle$ CPU (9) [s] $\rangle$ & $\langle$ CPU (10) [s] $\rangle$ \\
\hline 10 & 0.90 & 1.00 & 1.97 & 1.35 & 0.59 & 1.00 \\
15 & 0.59 & 0.65 & 1.26 & 0.87 & 1.08 & 2.45 \\
20 & 0.48 & 0.52 & 1.01 & 0.67 & 3.04 & 7.55 \\
25 & 0.48 & 0.53 & 1.00 & 0.65 & 8.20 & 20.69 \\
30 & 0.41 & 0.45 & 0.84 & 0.54 & 20.33 & 50.89 \\
\hline
\end{tabular}

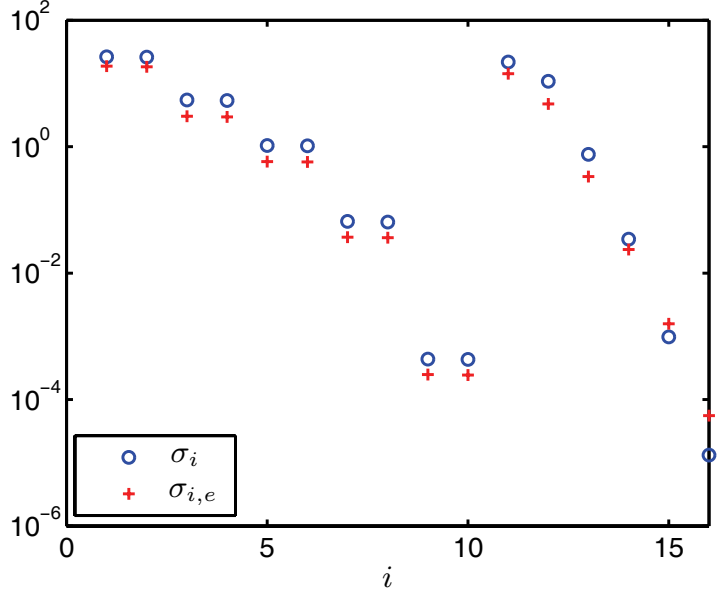

Fig. 1. The Hankel singular values for the case $k=0.05$.

using the generalized Gramians $P^{1}, Q^{1}$ and $P^{2}, Q^{2}$. This preserves the topological structure, and the regular a priori error bound on $\left\|T\left(k, G^{1}, G^{2}\right)-T\left(k, \hat{G}^{1}, \hat{G}^{2}\right)\right\|_{\infty}$ holds, see [14]. However, the LMIs (12) are not always feasible. Actually, feasibility of the LMIs is equivalent to the feedback system being strongly stable, see [13]. An alternative is to apply extended balanced truncation. Since there are more degrees of freedom in extended Gramians than in generalized Gramians, there is a higher chance to find Gramians with the right block-diagonal structure. Instead of solving (12), it is therefore suggested that the LMIs

$\min \operatorname{Tr} R$ and $\min \operatorname{Tr} S$; subject to (5)-(6) and

$$
R=R^{T}=\operatorname{diag}\left\{R^{1}, R^{2}\right\}, S=S^{T}=\operatorname{diag}\left\{S^{1}, S^{2}\right\},
$$

where $R^{k}, S^{k} \in \mathbb{R}^{n^{k} \times n^{k}}, k=1,2$, are solved. Note that it is only on the $R, S$ components that we need to enforce the block-diagonal constraint, since these are the components used for balancing. The variables $P, Q$ are full matrices. It is clear that if there are solutions to (12), then there are solutions to (13).

The subsystem with transfer function $G^{1}(z)$ is in this example a 10-th order highly resonant stable system, $n^{1}=10$, and $G^{2}(z)$ is a 6-th order stable low-pass system, $n^{2}=6$. For $0 \leq k \leq 0.16$, the feedback system $T\left(k, G^{1}, G^{2}\right)$ is stable. The LMIs (12) are feasible for $0 \leq k \leq 0.09$, and the LMIs (13) are feasible for $0 \leq k \leq 0.11$. Hence, extended balanced truncation is more often applicable, as expected. The generalized and the extended Hankel singular values are plotted in Fig. 1 for the case $k=0.05$. The first ten values correspond to the states in $G^{1}$, and the last six values correspond to states in $G^{2}$. The values indicate how important the states in the subsystems are with respect to the closed-loop mapping $T\left(k, G^{1}, G^{2}\right)$. One can use the values together with the bound in Theorem 2 to choose model orders $r^{1}, r^{2}$, given an acceptable approximation error. Here, we just truncate states that correspond to values of magnitude less than one, for simplicity. This gives $r^{1}=4$ and $r^{2}=2$, a reduction with 10 states in total. Generally, it is seen

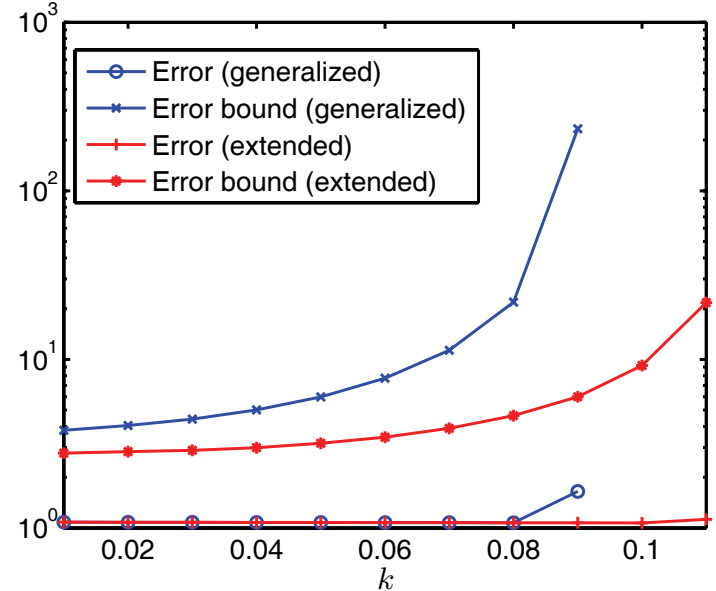

Fig. 2. The states corresponding to $i=5-10$ (in $G^{1}$ ) and $i=13-16$ (in $G^{2}$ ) are truncated. Extended balanced truncation gives slightly better approximations than generalized balanced truncation, gives better error bounds, and works for larger $k$.

that the extended values are smaller than the generalized ones, but not always (see $i=15,16$ ). The reason for this is that minimizing the traces of $P, R, Q, S$ as done in (12)-(13) is only a heuristic to make the singular values small.

In Fig. 2, it is seen that extended balanced truncation gives slightly better approximations than generalized balanced truncation for all feedback gains $k$, and the obtained error bounds are much better. If the approximation orders $r^{1}, r^{2}$ are changed, similar results are obtained. The main benefit with extended balanced truncation is that it can be applied more often (larger $k$ ), that the error bounds are tighter just as in Example 1, and that the extended inequalities are numerically better behaved with block-diagonal constraints.

\section{CONCLUSions}

An extension to the balanced-truncation method has been presented. Extended Gramians with at least twice as many variables as normal and generalized Gramians were defined. It was shown that the extra variables can be used when one wants to preserve topological constraints and to improve a priori error bounds in model reduction. The price for this is an increased computation time. An interesting problem for future research is to find better optimization objective functions that better exploit the additional freedom given by the extended Gramians.

\section{Acknowledgment}

The author would like to thank Prof. R. Smith for bringing his attention to the papers [15], [16], and Dr. G. Kotsalis for helpful discussions.

\section{REFERENCES}

[1] C. Mullis and R. Roberts, "Synthesis of minimum roundoff noise fixed point digital filters," IEEE Transactions on Circuits and Systems, vol. 23, pp. 551-562, 1976. 
[2] B. C. Moore, "Principal component analysis in linear systems: controllability, observability, and model reduction," IEEE Transactions on Automatic Control, vol. 26, no. 1, pp. 17-32, February 1981.

[3] L. Pernebo and L. Silverman, "Model reduction via balanced state space representation," IEEE Transactions on Automatic Control, vol. 27, pp. 382-387, 1982.

[4] D. Enns, "Model reduction with balanced realizations: an error bound and a frequency weighted generalization," in Proceedings of the IEEE Conference on Decision and Control, Las Vegas, Nevada, 1984.

[5] K. Glover, "All optimal Hankel-norm approximations of linear multivariable systems and their $L_{\infty}$-error bounds," International Journal of Control, vol. 39, pp. 1115-1193, 1984.

[6] U. Al-Saggaf and G. Franklin, "An error-bound for a discrete reducedorder model of a linear multivariable system," IEEE Transactions on Automatic Control, vol. 32, no. 9, pp. 815-819, 1987.

[7] D. Hinrichsen and A. Pritchard, "An improved error estimate for reduced-order models of discrete-time systems," IEEE Transactions on Automatic Control, vol. 35, no. 3, pp. 317-320, 1990.

[8] D. Kavranoğlu and M. Bettayeb, "Characterization of the solution to the optimal $H_{\infty}$ model reduction problem," Systems \& Control Letters, vol. 20, pp. 99-107, 1993.

[9] K. M. Grigoriadis, "Optimal $H_{\infty}$ model reduction via linear matrix inequalities: continuous- and discrete-time cases," Systems \& Control Letters, vol. 26, pp. 321-333, 1995.

[10] G. Dullerud and F. Paganini, A course in robust control theory - a convex approach. Springer-Verlag, 2000.

[11] C. Beck, J. Doyle, and K. Glover, "Model reduction of multidimensional and uncertain systems," IEEE Transactions on Automatic Control, vol. 41, no. 10, pp. 1466-1477, October 1996.

[12] L. Li and F. Paganini, "Structured coprime factor model reduction based on LMIs," Automatica, vol. 41, no. 1, pp. 145-151, Jan. 2005.

[13] C. Beck, "Coprime factors reduction methods for linear parameter varying and uncertain systems," Systems \& Control Letters, vol. 55, pp. 199-213, 2006.

[14] H. Sandberg and R. M. Murray, "Model reduction of interconnected linear systems," Optimal Control Applications and Methods, vol. 30, no. 3, pp. 225-245, May/June 2009, special issue: Directions, applications and methods in robust control.

[15] M. De Oliviera, J. Bernussou, and J. Geromel, "A new discrete-time robust stability condition," Systems \& Control Letters, vol. 37, pp. 261265, 1999.

[16] M. De Oliviera, J. Geromel, and J. Bernussou, "Extended $H_{2}$ and $H_{\infty}$ norm characterizations and controller parametrizations for discrete-time systems," International Journal of Control, vol. 75, no. 9, pp. 666-679, 2002.

[17] M. V. Subbotin and R. S. Smith, "Design of distributed decentralized estimators for formations with fixed and stochastic communication topologies," Automatica, vol. 45, pp. 2491-2501, 2009.

[18] H. Sandberg, "Model reduction of linear systems using extended balanced truncation," in Proceedings of the 2008 American Control Conference, Seattle, Washington, June 2008, pp. 4654-4659.

[19] J. Sturm, "Using SeDuMi 1.02, a MATLAB toolbox for optimization over symmetric cones," Optimization Methods and Software, vol. 1112, pp. 625-653, 1999.

[20] P. Bendotti and C. Beck, "On the role of LFT model reduction methods in robust controller synthesis for a pressurized water reactor," IEEE Transactions on Control Systems Technology, vol. 7, no. 2, pp. 248257, March 1999.

[21] S. Boyd, L. El Ghaoui, E. Feron, and V. Balakrishnan, Linear Matrix Inequalities in System and Control Theory. Society for Industrial and Applied Mathematics, 1994.

[22] H. Sandberg and A. Rantzer, "Balanced truncation of linear time-varying systems," IEEE Transactions on Automatic Control, vol. 49, no. 2, pp. 217-229, Feb. 2004.

[23] A. Vandendorpe and P. Van Dooren, Model Order Reduction: Theory, Research Aspects and Applications, ser. Mathematics in Industry Series. Springer Verlag, 2007, ch. Model reduction of interconnected systems. 